\title{
MapLearn: uma abordagem para apoiar a elaboração de cenários de aprendizagem baseado em personas
}

\author{
Eline F. de A. Welter ${ }^{1}$, Luciana A. M. Zaina ${ }^{2}$, Renato F. L. dos Santos ${ }^{1}$ \\ ${ }^{1}$ Departamento de Computação - Instituto Federal de Ciência e Tecnologia \\ de São Paulo (IFSP) Campus Itapetininga - Av. João O. de Oliveira, 1561, \\ V1. Asem - 18202-000 - Itapetininga - SP - Brasil \\ ${ }^{2}$ Departamento de Computação - Universidade Federal de São Carlos \\ (UFSCAR) - Campus Sorocaba - Rod. João L. dos Santos, Km \\ 110 - SP-264 - 18052-780 - Sorocaba - SP - Brasil \\ \{elinewelter, renato.itp\}@ifsp.edu.br, lzaina@ufscar.br
}

\begin{abstract}
This article presents an approach to support the teacher in the development of learning scenarios focusing on the perception of student's profile. For this, the approach takes into account the concept of personas adapting the use of empathy maps to the context of e-learning. A case study with teachers was conducted to validate the approach. The proposal brings contributions to guide teacher in the use of electronic learning objects and environments considering student's profile.
\end{abstract}

Resumo. Este artigo apresenta uma abordagem para apoiar o professor na elaboração de cenários de aprendizagem centrando-se na percepção sobre o perfil dos alunos. Para isto, a abordagem apoiou-se no fundamento de personas adaptando o uso de mapas de empatia para o contexto de aprendizagem eletrônica. Um estudos de caso com professores foi conduzido para validação da abordagem. A proposta traz contribuições para guiar o professor no uso e criação de ambientes e objetos de aprendizagem eletrônica considerando o perfil dos alunos.

\section{Introdução}

Um grande desafio vivido pela área de educação tem sido unir a crescente imersão dos jovens na tecnologia com a utilização do meio tecnológico como ferramenta de apoio ao desenvolvimento da aprendizagem. Observa-se que há um constante crescimento no uso da tecnologia tanto por parte dos professores quanto dos alunos. Segundo dados da pesquisa TIC Educação 2012, realizada pelo Comitê Gestor da Internet no Brasil, o computador já é uma ferramenta presente em todas as 856 instituições entrevistadas e, entre elas, 74\% contam também com computadores portáteis [Cetic 2012]. A pesquisa também aponta que $69 \%$ dos alunos brasileiros e $86 \%$ dos professores utilizam a Internet todos os dias; que $50 \%$ das atividades mais comuns em laboratório de informática são atividades de pesquisa em revistas ou livros, $33 \%$ são para a realização de jogos educativos, e $32 \%$ para prática de exercícios sobre o conteúdo exposto em aula.

Embora muitas aplicações tenham sido desenvolvidas para dar suporte as atividades educativas através de computadores (aprendizagem eletrônica), ainda há uma lacuna 
VI Congresso Brasileiro de Informática na Educação (CBIE 2017)

Anais do XXIII Workshop de Informática na Escola (WIE 2017)

por abordagens que auxiliem o professor a direcionar esforços sobre: quem de fato são os alunos que participarão da atividade que ele está planejando, que mídias ou objetos de aprendizagem são mais adequados, entre outras informações. Ou seja, em como concentrar seu esforços para utilizar os ambientes virtuais de forma a potencializar o uso do ambiente de acordo com o perfil dos alunos que está trabalhando. Durante a elaboração de suas atividades virtuais o professor concentra-se em planejar um ou mais cenários de aprendizagem que sejam aplicados ao conteúdo que será abordado. Cenários de aprendizagem são definidos a partir de um conjunto de elementos interativos como vídeos, figuras, jogos, textos, simuladores, entre outros que, sinergicamente atendem um objetivo de aprendizagem [Zaina et al. 2012].

A percepção sobre o perfil do aluno tem sido abordada em trabalhos que buscam adaptar o ambiente de aprendizagem ao aluno de forma individualizada através de estilos de aprendizagem [Aguiar et al. 2015] [Aguiar et al. 2014] [Carvalho et al. 2014] [Santos 2013] [Zaina and Bressan 2008]. Embora tal foco seja de extrema relevância dentro do contexto de aprendizagem eletrônica, muitos ainda são os ambientes que não possuem tal adaptação. Desta forma, é de suma importância que o professor tivesse uma ferramenta de apoio que o permitisse agrupar a visão das características mais determinantes do grupo de alunos que está trabalhando. E, a partir desta visão, pudesse realizar escolhas sobre os elementos ou o ambiente virtual que desejaria utilizar para atingir um dado objetivo de aprendizagem.

A percepção é centrada no perfil, mas busca salientar as características mais determinantes de um grupo de pessoas, e esta percepção é foco da área de Experiência do Usuário (UX - User eXperience). UX preocupa-se em entender como um produto é utilizado por pessoas [Rogers et al. 2013]. E um dos fundamentos que auxiliam nessa percepção são as personas, que é uma técnica que descreve as características que representem um grupo de pessoas a partir da criação de personagens fictícios. Diversos modelos para auxiliar a criação e descrição das personas tem sido utilizados. Um deles são os mapas de empatia, que buscam adicionar a descrição da persona os aspectos sobre o ambiente em que o produto será utilizado e as emoções envolvidas.

Este artigo tem como objetivo apresentar uma abordagem para apoiar o professor na elaboração de cenários de aprendizagem, centrando-se na percepção sobre o perfil de um grupo de alunos. Para isto, a abordagem proposta apoiou-se no fundamento de personas, adaptando o uso de mapas de empatia para o contexto de aprendizagem eletrônica. Acredita-se que a proposta possa contribuir como uma ferramenta guia para o professor, no uso de diferentes ambientes de aprendizagem eletrônica. Para validação da proposta foi conduzido um estudo de caso com professores, visando verificar o uso e aceitação da técnica proposta.

\section{Referencial Teórico e Trabalhos Relacionados}

A proposta deste trabalho se baseia no fundamento de construção de personas como suporte ao professor para elaboração de seus cenários de aprendizagem, apoiado pelos mapas de empatia.

Uma persona auxilia a personificar uma ideia abstrata de um grupo de possíveis usuários, identificando seus atributos mais relevantes em um dado contexto [Caballero et al. 2014]. A criação de uma persona pode ser feita através de observação, 
entrevista ou aplicação de questionário. Contudo, muitas vezes o que se utiliza é o conhecimento prévio sobre o usuário, que os próprios especialistas da área possuem [Billestrup et al. 2014, Dittmar and Hensch 2015]. Considerando o contexto educacional, o professor é considerado um especialista, pois possui um conhecimento prévio do perfil dos alunos. Em um trabalho sobre desenvolvimento de jogos [Moser 2012] relata que personas podem ser utilizadas em diferentes cenários de desenvolvimento e que no desenvolvimento de jogos educacionais a criação de personas é um artefato essencial para que os desenvolvedores tenham a mesma visão do usuário-alvo, sem esquecer suas necessidades e habilidades.

Considerando o perfil dos alunos, um conceito utilizado é de perfil de aprendizagem. O perfil de aprendizagem é determinado através de características predominantes, denominadas de estilos de aprendizagem, que determinam as estratégias utilizadas no processo de ensino-aprendizagem [Aguiar et al. 2014]. Alguns trabalhos como [Carvalho et al. 2014, Aguiar et al. 2015, Zaina et al. 2012], buscam utilizar o perfil de aprendizagem como característica determinante para adaptação do ambiente virtual de aprendizagem. O trabalho proposto neste artigo usa o perfil de aprendizagem como direcionador para o professor durante a escolha dos elementos que comporão o cenário de aprendizagem.

Já os mapas de empatia consistem em uma técnica que estimula o outro a colocarse no lugar do usuário e ver o mundo através das suas perspectivas [Bratsberg 2012, Knox 2014]. Pode ser considerada uma forma de descrever personas a partir de aspectos que incluem o emocional. Atualmente, oito aspectos compõem um mapa de empatia e buscam descrever o perfil do usuário: Vê, Fala e Faz, Pensa e Sente, Ouve, Necessidades e Dores [Bland 2012]. No contexto de aprendizagem eletrônica a técnica pode auxiliar tanto um desenvolvedor a projetar as aplicações quanto um professor a potencializar o uso dos ambientes virtuais como apoio à aprendizagem.

\section{MapLearn: Abordagem Proposta}

Baseando-se nos fundamentos de mapa de empatia e considerando a lacuna por ferramentas que auxiliem o professor a direcionar esforços que potencializem o uso dos ambiente virtuais, este artigo tem como objetivo apresentar uma abordagem denominada MapLearn. A proposta visa apoiar o professor na elaboração de cenários de aprendizagem centrando-se na percepção sobre o perfil dos alunos através da elaboração de mapas de empatia que delineie seu público-alvo.

O MapLearn é composto de três artefatos: (i) o mapa de empatia e sua descrição, (ii) cartões que descrevem o perfil de aprendizagem, e (iii) um guia que direciona o professor na construção do mapa. Um ponto importante é que uma vez elaborado o mapa, este pode ser reutilizado alterando-se alguns dos campos ou até mesmo pode ser compartilhado entre docentes que trabalham com a mesma turma.

O mapa de empatia foi adaptado da proposta original [Bland 2012] incluindo-se características que fossem aderentes ao contexto de aprendizagem eletrônica (Figura 1). Foram retirados campos e adicionados novos de forma que contemplassem as habilidades relacionadas ao grupo de alunos, em que tipos de dispositivos os alunos poderiam utilizar o cenário de aprendizagem projetado, que canais de interação usaria (texto, imagens, voz, vídeos, jogos, etc), objetivo da atividade e perfil de aprendizagem que o professor 
VI Congresso Brasileiro de Informática na Educação (CBIE 2017)

Anais do XXIII Workshop de Informática na Escola (WIE 2017)

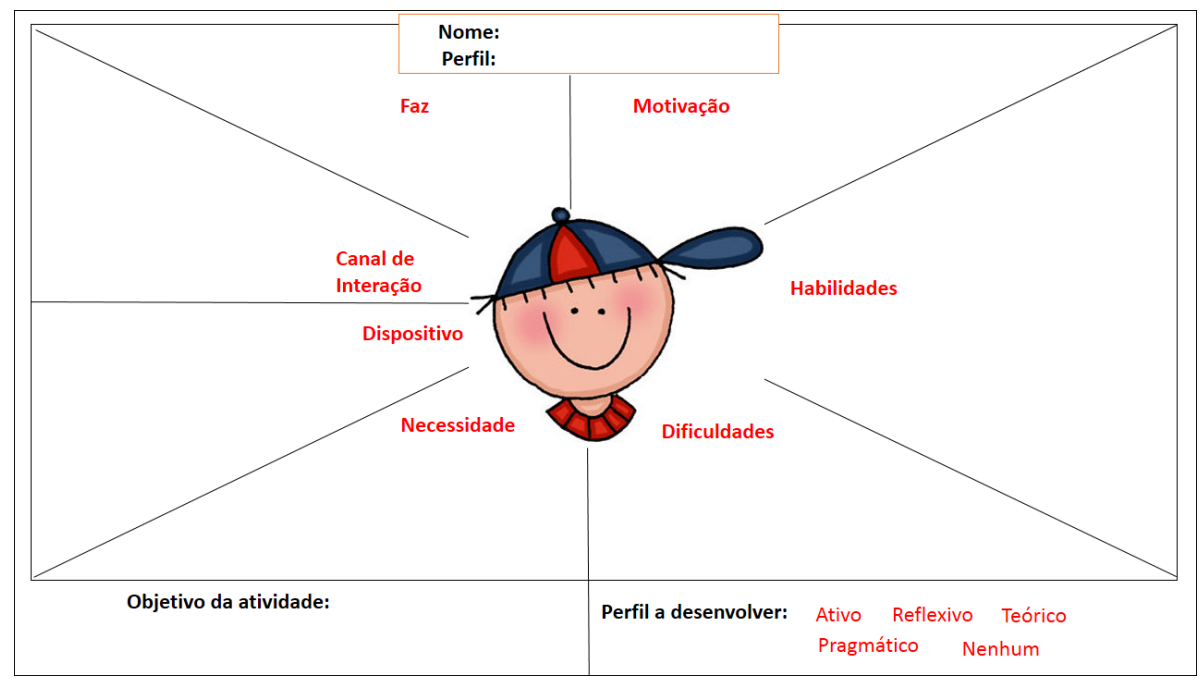

Figura 1. Modelo do mapa de empatia
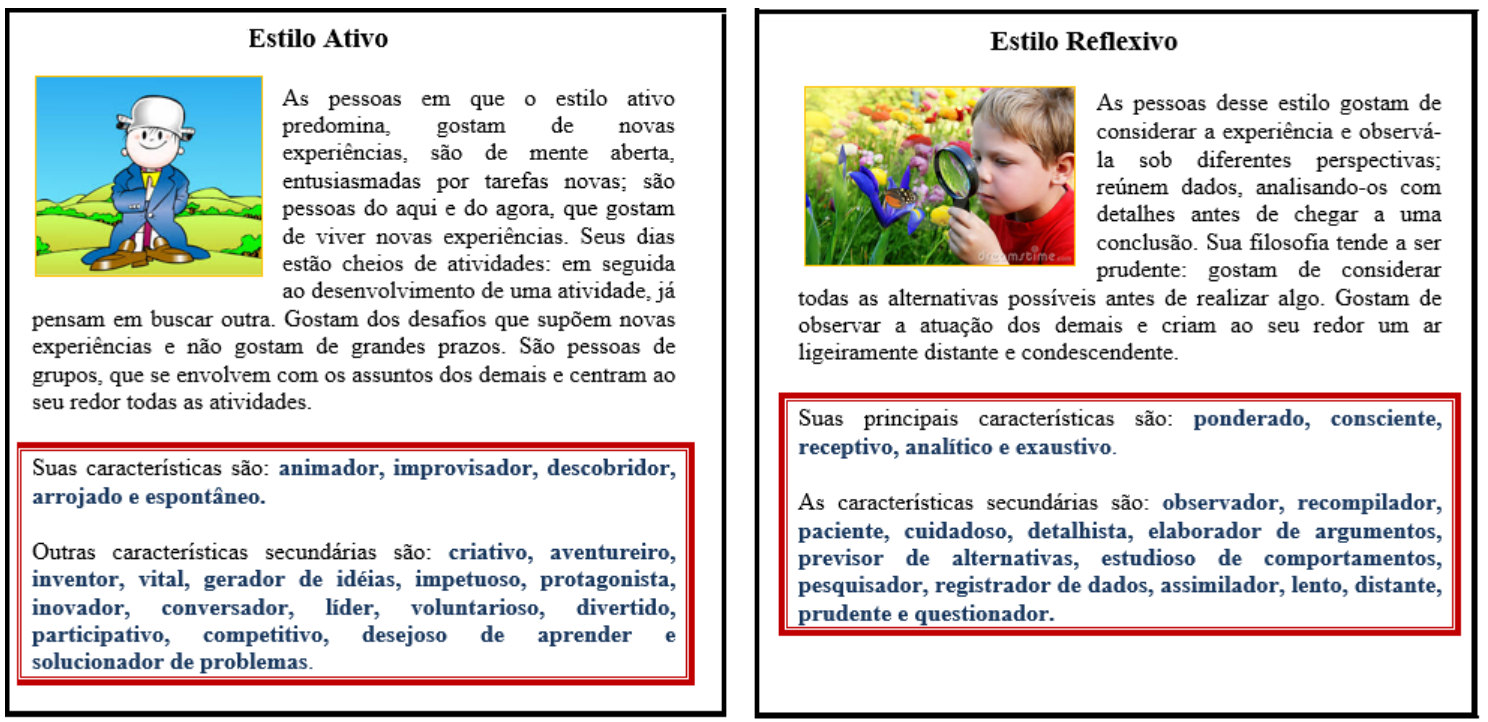

\section{Figura 2. Cartões com perfis de Ativo e Reflexivo baseado no trabalho de [Barros 2014]}

gostaria de desenvolver nos alunos, entre outros.

Os cartões com os perfis de aprendizagem (Figura 2) são artefatos de apoio para auxiliar o professor a identificar qual é o perfil da persona que será construída, os cartões descrevem os perfis de aprendizagem e são adaptáveis ao modelo utilizado. Há diversos perfis catalogados porém, para este estudo foi escolhido o modelo de perfis defendido por Honey e Munford [Honey and Mumford 2006], pois o mesmo já havia sido utilizado em outro experimento com um grupo de professores do ensino Fundamental II [Barros 2014].

A elaboração do mapa de empatia é suportada por um guia direcionador composto por: (a) descrição e sugestão de quais informações cada campo deve receber (Tabela 1); e (b) questões-guias (Tabela 2 ) - conjunto de questões que auxiliam o professor a preencher cada campo do mapa - nem todas as questões necessitam ser preenchidas. 
VI Congresso Brasileiro de Informática na Educação (CBIE 2017)

Anais do XXIII Workshop de Informática na Escola (WIE 2017)

Tabela 1. Descrição dos campos do MapLearn

\begin{tabular}{l|l}
\hline \hline \multicolumn{1}{c|}{ Campo } & \multicolumn{1}{c}{ Descrição } \\
\hline Nome & Nome fictício para a identificação do MapLearn. \\
\hline Perfil & Perfil de aprendizagem identificado. \\
\hline Faz & $\begin{array}{l}\text { Quais as características da rotina do aluno, o que ele faz, quais as atividades que mais } \\
\text { gosta e não gosta, quais são seus hobbies. }\end{array}$ \\
\hline Motivação & $\begin{array}{l}\text { Quais motivações que o aluno possui, como por exemplo, o que o estimula, se gosta de } \\
\text { ser desafiado ou se gosta de utilizar tecnologia no seu cotidiano. }\end{array}$ \\
\hline Habilidades & $\begin{array}{l}\text { Quais habilidades que o aluno possui, se tem facilidade em alguma tarefa, se possui } \\
\text { habilidades em utilizar ferramentas tecnológicas, etc. }\end{array}$ \\
\hline Dificuldades & Possui dificuldade em alguma atividade, matéria ou quais problemas enfrenta. \\
\hline Necessidades & $\begin{array}{l}\text { Necessidades que o aluno possui ou o que precisa ser melhorado de acordo com as } \\
\text { dificuldades apontadas. }\end{array}$ \\
\hline $\begin{array}{l}\text { Dispositivos } \\
\text { Quais os dispositivos tecnológicos (celular, tablet ou computador) o aluno possui maior } \\
\text { habilidade, o que mais gosta ou que possui acesso. }\end{array}$ \\
\hline $\begin{array}{l}\text { Canais } \\
\text { interação }\end{array}$ & $\begin{array}{l}\text { Quais tipos de objetos (mídias) serão utilizadas com os alunos, textos, imagens (fotos, } \\
\text { gráficos, mapas), som (narração), desafios ou outros. }\end{array}$ \\
\hline $\begin{array}{l}\text { Objetivos da } \\
\text { Atividade }\end{array}$ & Qual será o objetivo da aula que será desenvolvida para este MapLearn. \\
\hline $\begin{array}{l}\text { Perfis a } \\
\text { desenvolver }\end{array}$ & Qual perfil de aprendizagem gostaria de trabalhar. \\
\hline \hline
\end{tabular}

Tabela 2. Questões do Guia para elaborar o mapa

\begin{tabular}{l|l}
\hline \hline Campo & Questão-Guia \\
\hline Faz & $\begin{array}{l}\text { (Q1) O que o aluno faz?; (Q2) Atividades que mais gosta?; (Q3) Atividades que NÃO } \\
\text { gosta?; (Q4) Quais são seus hobbies? }\end{array}$ \\
\hline Motivação & (Q5) O que estimula o aluno?; (Q6) Gosta de desafios?; (Q7) Gosta de tecnologia? \\
\hline Habilidades & $\begin{array}{l}\text { (Q8) Em quais atividades o aluno é bom?; (Q9) Disciplinas que possui facilidade?; (Q10) } \\
\text { Gosta de leitura?; (Q11) Facilidade em utilizar tecnologia? }\end{array}$ \\
\hline Necessidades & (Q12) Quais as dificuldades que precisa trabalhar para melhorar? \\
\hline Dispositivos & (Q13) Utiliza celular, tablet ou computador?; (Q14) Qual o dispositivo de preferência? \\
\hline $\begin{array}{l}\text { Canal } \\
\text { interação }\end{array}$ & $\begin{array}{l}\text { (Q15) Tem preferência por visualizar figuras?; (Q16) Tem preferência por leitura?; (Q17) } \\
\text { Tem preferência por jogos?; (Q18) Tem preferência por ouvir descrições? }\end{array}$ \\
\hline \hline
\end{tabular}

\section{Validação da Proposta}

Para validação da proposta foi aplicado um estudo de caso com professores, sendo que, os participantes autorizaram, através de um TCLE (Termo de Consentimento Livre e Esclarecido), a coleta e uso dos dados. As subseções a seguir descrevem o estudo de caso e a discussão dos resultados.

\subsection{Estudo de Caso}

O objetivo deste estudo de caso foi verificar o uso e aceitação do MapLearn como ferramenta de apoio à elaboração de cenários ou objetos de aprendizagem, centrando-se na percepção sobre o perfil de um grupo de alunos. O estudo foi realizado entre o final de 2015 e início de 2016 e contou com a participação de 20 professores que atuavam no Ensino Fundamental II e Médio da rede pública de ensino da cidade de Itapetininga. Os professores foram convidados a participar através de convite aberto às instituições de ensino, e não possuíam conhecimentos prévios sobre uso de mapas de empatia ou personas.

Como ferramentas de apoio a coleta de dados, foi elaborado um formulário com o propósito de identificar o perfil dos professores participantes e coletar a percepção dos 
VI Congresso Brasileiro de Informática na Educação (CBIE 2017)

Anais do XXIII Workshop de Informática na Escola (WIE 2017)

participantes sobre a (1) facilidade de compreensão, (2) facilidade de uso e (3) utilidade da técnica proposta. As questões (Tabela 3) foram adaptadas do modelo de aceitação de tecnologia TAM (Technology Acceptance Model), que possibilita avaliar a aceitação de uma ferramenta tecnológica sob o ponto de vista de quem irá utilizá-la [Venkatesh et al. 2003]. Os participantes assinalaram a resposta que melhor representasse sua opinião, sendo que cada questão foi estruturada através da escala Likert de seis pontos, partindo do ponto 'Discordo Totalmente' até 'Concordo Totalmente'. Outro artefato de coleta que foi preparado visava verificar a utilidade das questões-guia (Tabela 2) como apoio à elaboração do mapa. Os participantes deveriam assinalar para cada questão-guia, se a mesma foi utilizada por ele e se na visão dele, esta possuía ou não relevância na elaboração do mapa.

Tabela 3. Afirmativas sobre o MapLearn

\begin{tabular}{l|l}
\hline \hline Aspecto & Afirmativa \\
\hline \multirow{2}{*}{$\begin{array}{l}\text { Facilidade } \\
\text { de uso }\end{array}$} & A1. Foi fácil construir a persona que represente a turma com uso do MapLearn. \\
\cline { 2 - 2 } & A2. Foi fácil aprender a usar o MapLearn. \\
\cline { 2 - 2 } & A3. As questões guia ajudou na construção do mapa. \\
\cline { 2 - 2 } & A4. Após aprender a utilizar, foi fácil ganhar habilidade para usar o MapLearn. \\
\cline { 2 - 2 } & A5. Consegui utilizar a técnica como eu desejava. \\
\hline \multirow{2}{*}{$\begin{array}{l}\text { Facilidade de } \\
\text { compreensão }\end{array}$} & A6. Considero fácil lembrar como usa o MapLearn. \\
\hline \multirow{2}{*}{$\begin{array}{l}\text { Utilidade da } \\
\text { técnica }\end{array}$} & A7. Usar o MapLearn pode melhorar a forma como eu preparo as aulas. \\
\cline { 2 - 2 } & A8. Considero o MapLearn útil para a preparação de aulas. \\
\cline { 2 - 2 } & A9. Utilizaria o MapLearn com frequência. \\
\hline \hline
\end{tabular}

A condução do estudo de caso teve duração de 40 minutos. Inicialmente foi apresentado o objetivo da atividade e a técnica do MapLearn, com a execução de um breve exemplo para aquecimento. Durante a condução, haviam dois observadores realizando anotações e esclarecendo dúvidas dos participantes.

Solicitou-se aos participantes que definissem: um tema de estudo e escolhessem uma de suas turmas em que o objeto ou cenário de aprendizagem seria possivelmente utilizado. Na sequência os professores receberam os artefatos do MapLearn: mapa de empatia, as descrições do mapa , as questões-guias e cartões com os perfis de aprendizagem e iniciaram a criação da persona, como mostrado na Figura 3

\subsection{Análise dos dados coletados}

O perfil dos participantes era de 50\% entre 31 e 45 anos de idade, $45 \%$ até 30 anos e os demais acima de 46 anos. Todos possuíam uma frequência alta em relação ao uso de computadores durante a semana com $65 \%$ fazendo uso mais de 4 vezes por semana, sugerindo que os professores têm contato frequente com a tecnologia (Figura 4).

Analisando o formulário sobre a aceitação da proposta na percepção dos participantes (respondido ao final da atividade), obteve-se os resultados: (i) facilidade de compreensão do uso da técnica teve $80 \%$ de aceitação; (ii) facilidade no uso da técnica apresentou $90,67 \%$ de aceitação; e (iii) utilidade da técnica apresentou 88,33\% de grau de concordância (Figura 5). Tal resultado demonstra que na percepção dos participantes, a técnica do MapLearn é útil e aplicável. As Figuras 6 e 7 apresentam os resultados organizados por questão avaliada. 
VI Congresso Brasileiro de Informática na Educação (CBIE 2017)

Anais do XXIII Workshop de Informática na Escola (WIE 2017)
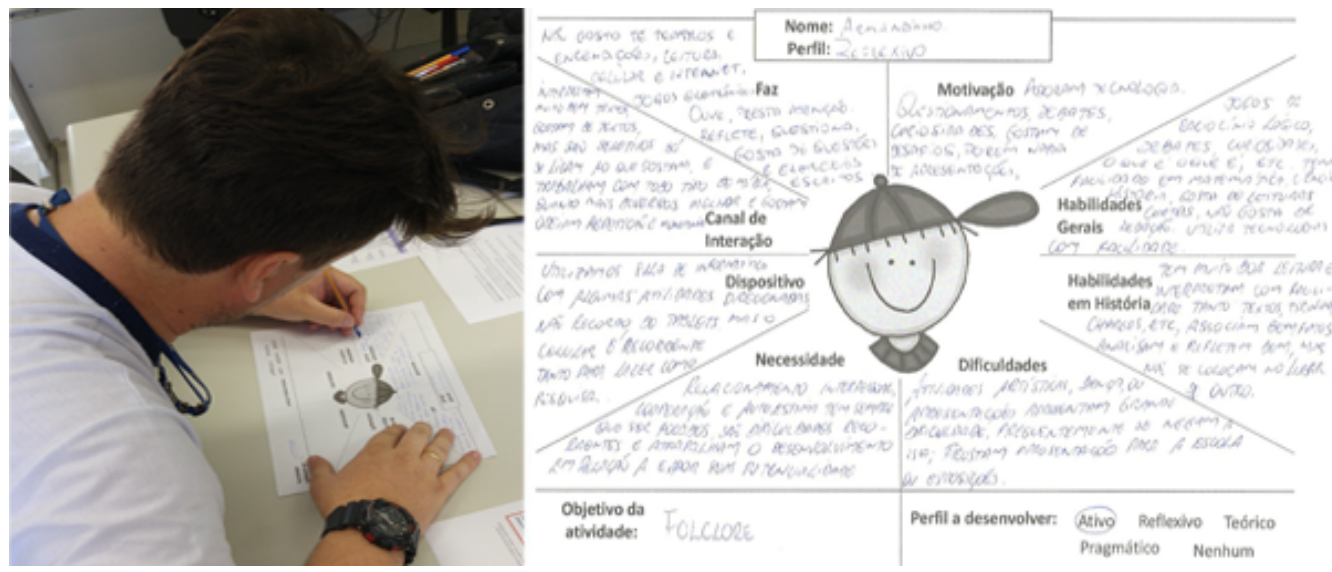

Figura 3. Atividade de uso do MapLearn

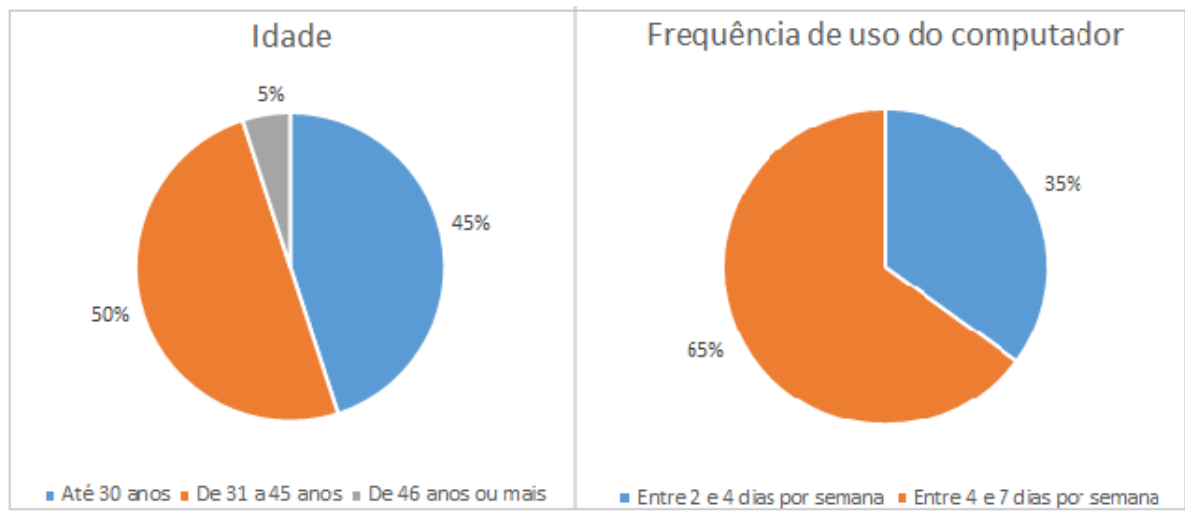

(a)

(b)

Figura 4. Idade e Frequência de uso de computador pelos participante

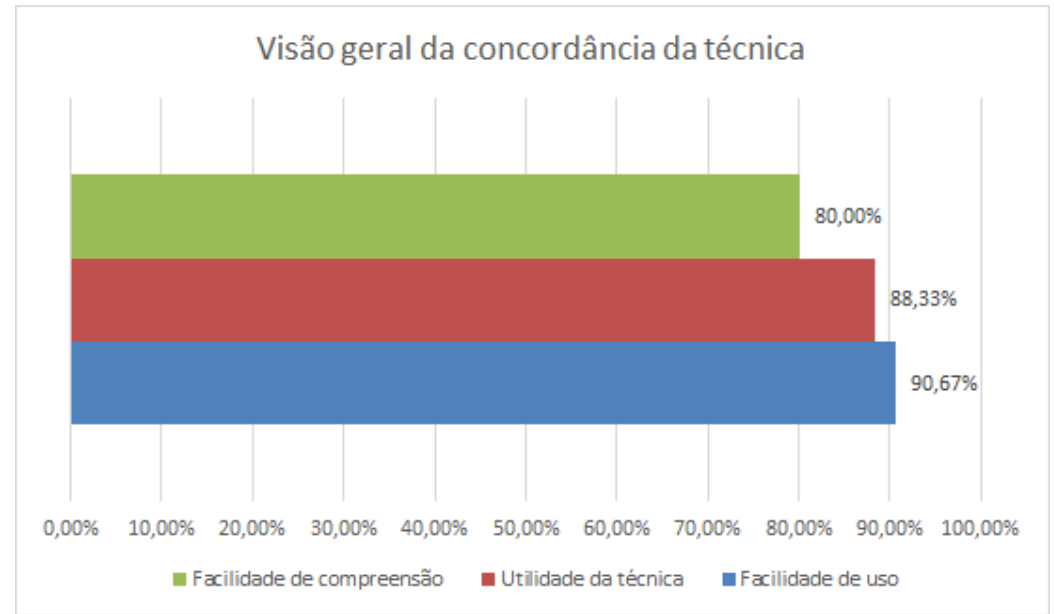

Figura 5. Visão geral do grau de concordância sobre o MapLearn

Observando o uso das questões-guia, os pesquisadores verificaram que todos os participantes as utilizaram durante a elaboração do mapa. As questões também foram avaliadas pelos participantes que apontaram se a mesma foi ou não utilizada e se possuía relevância ou não para o preenchimento dos campos do mapa. Considerando as questões 
VI Congresso Brasileiro de Informática na Educação (CBIE 2017)

Anais do XXIII Workshop de Informática na Escola (WIE 2017)

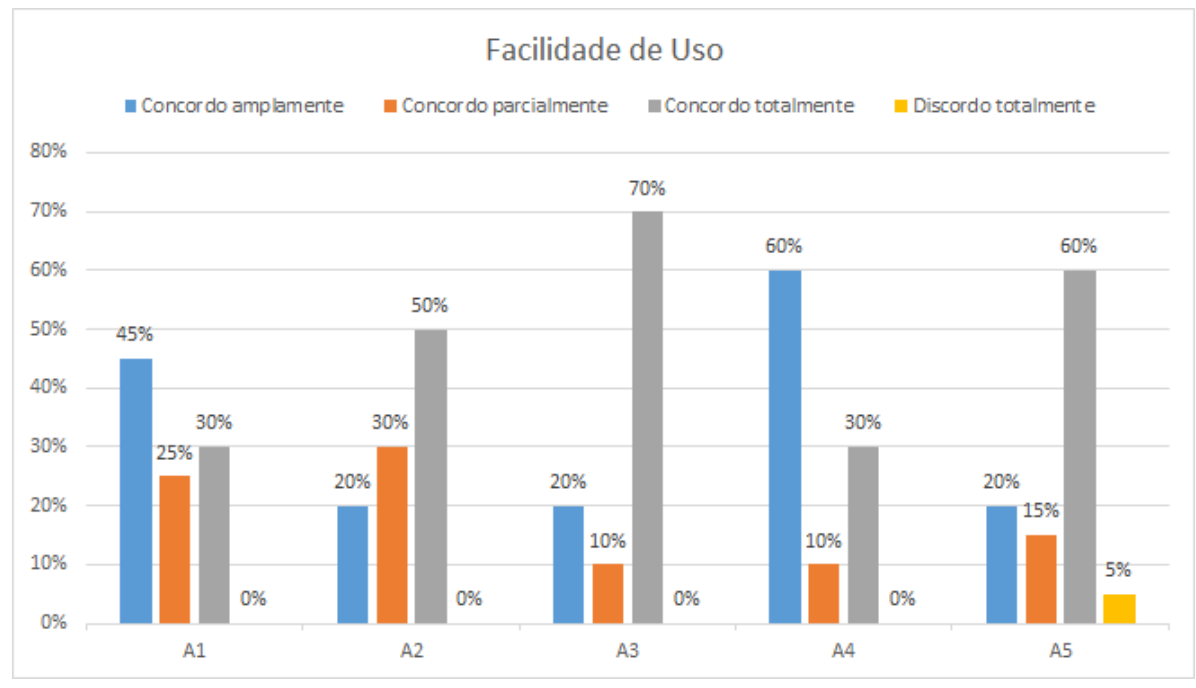

Figura 6. Facilidade de uso do MapLearn

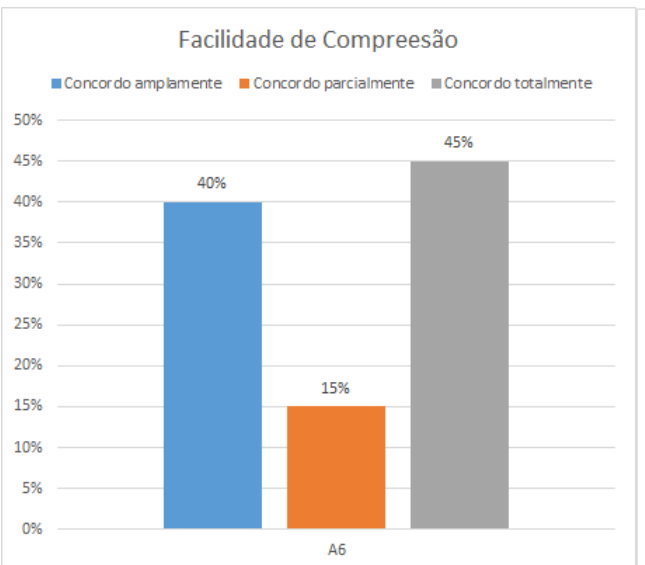

(a)

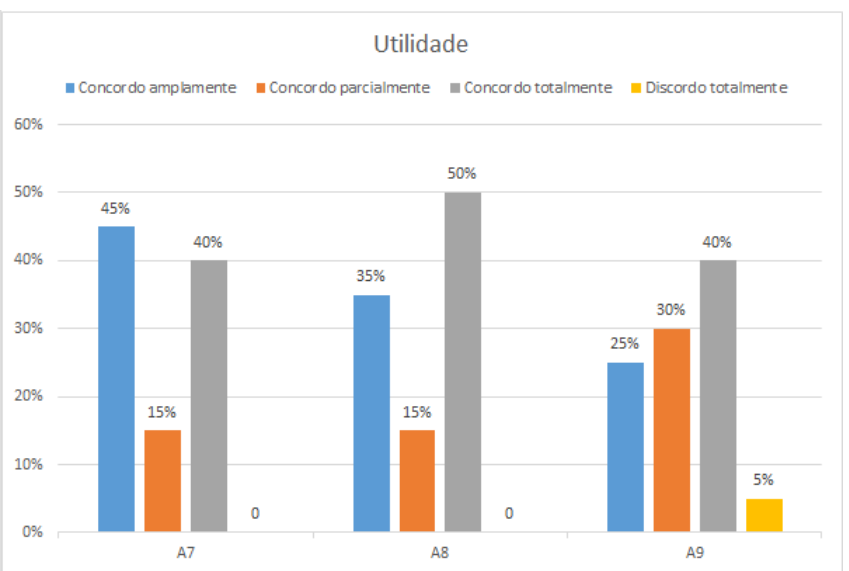

(b)

Figura 7. Facilidade de compreensão e utilidade do MapLearn

marcadas como 'Não Usada' e 'Não Relevante' obteve-se algumas justificativas dos participantes, que podem ser resumidas em: (a) Q4 - utilizada por apenas 40\% dos professores; porém apesar da pouca utilização apenas $10 \%$ apontaram a questão como 'não relevante', como justificativa por não ter utilizado a questão, os participantes relataram desconhecer essa informação da turma; e (b) Q16 - não foi utilizada por 10\% dos professores e também 10\% marcaram como uma questão 'não relevante' pois, na visão dos participantes a questão era redundante em relação a Q10. Estes dois apontamentos serão considerados pelos pesquisadores para serem reavaliados, podendo ser reestruturados ou retirados em uma futura versão do MapLearn. As demais questões não utilizadas, foram justificadas pelos participantes como informações que eles desconheciam sobre a turma que foi idealizada porém, mesmo assim os professores marcaram as questões como relevantes.

Ao final da condução do estudo também foi realizada uma breve entrevista com os participantes sobre a experiência de uso da técnica. Estes apontaram boa aceitação da técnica e acreditam que há inúmeras possibilidades para a utilização do MapLearn em diferentes áreas de conhecimento. Além disto, muitos professores pediram cópias dos 
VI Congresso Brasileiro de Informática na Educação (CBIE 2017)

Anais do XXIII Workshop de Informática na Escola (WIE 2017)

artefatos para utilizarem em seu dia-a-dia na preparação de suas atividades.

\section{Conclusão e Trabalhos Futuros}

A abordagem proposta neste trabalho teve como objetivo apoiar o professor na elaboração de cenários de aprendizagem, buscando introduzir a visão da experiência do usuário através da técnica de personas. Para isto, os mapas de empatia foram adaptados para o contexto de aprendizagem eletrônica, criando-se artefatos que possibilitassem o uso da proposta pelo professor. Acredita-se que este trabalho traz contribuições importantes para guiar o professor durante o uso de ambientes de aprendizagem eletrônica. Através da abordagem o docente é conduzido a ter uma visão ampla do grupo de alunos que irá trabalhar e quais são os elementos do ambiente que podem ser utilizados para compor um cenário de aprendizagem. Os resultados obtidos através do estudo de caso demonstram a viabilidade e a boa aceitação no uso da abordagem. Como trabalho futuro, pretende-se realizar uma análise mais detalhada dos resultados obtidos, desenvolver uma ferramenta eletrônica para a criação do mapa de empatia e utilizar a proposta para criação de atividades em ambientes de aprendizagem virtual.

\section{Agradecimentos}

Agradecemos o apoio financeiro oferecido pela Fapesp a este projeto (processo $\mathrm{n}^{\mathrm{o}}$ 2013/25572-7), ao IFSP e Ufscar pelo apoio operacional e a todas as escolas e professores que prontamente colaboraram.

\section{Referências}

Aguiar, J. J., Fechine, J. M., and Costa, E. B. (2015). Recomendação de objetos de aprendizagem baseada na popularidade dos objetos e nos estilos de aprendizagem dos alunos. pages 1147-1156. CBIE-LACLO 2015. Anais do XXVI Simpósio Brasileiro de Informática na Educação (SBIE 2015).

Aguiar, J. J. B., Fechine, J. M., and Costa, E. B. (2014). Estilos cognitivos e estilos de aprendizagem em informática na educação: um mapeamento sistemático focado no sbie, wie e rbie. In Anais do XXV Simpósio Brasileiro de Informática na Educação, pages 441-450. SBC.

Barros, D. M. V. (2014). Estilos de aprendizagem e as tecnologias: Guias didáticos para o ensino fundamental. pages 301-314. Acessado: 21/10/2015.

Billestrup, J., Stage, J., Nielsen, L., and Kira, S. H. (2014). Persona Usage in Software Development: Advantages and Obstacles. ACHI 2014: The Seventh International Conference on Advances in Computer-Human Interactions Copyright (c) IARIA.

Bland, D. (2012). Agile coaching tip - what is an empathy map? http://www.bigvisible.com/2012/06/what-is-an-empathy-map/. Acessado: 5/3/2016.

Bratsberg, H. M. (2012). Empathy maps of the foursight preferences. Creative Studies Graduate Student Master's Project. Buffalo State College. Paper 176.

Caballero, L., Moreno, A. M., and Seffah, A. (2014). Persona as a tool to involving human in agile methods: contributions from hci and marketing. pages 283-290. 
VI Congresso Brasileiro de Informática na Educação (CBIE 2017)

Anais do XXIII Workshop de Informática na Escola (WIE 2017)

Carvalho, V. C., Dorça, F. A. Cattelan, R. G., and Araújo, R. D. (2014). Uma abordagem para recomendação automática e dinâmica de objetos de aprendizagem baseada em estilos de aprendizagem. In Anais do XXV Simpósio Brasileiro de Informática na Educação, pages 1188-1197.

Cetic (2012). Pesquisas e indicadores: Tic na educação. http://www.cetic.br/educacao/ 2012/alunos/index.htmlf. Acessado: 21/6/2015.

Dittmar, A. and Hensch, M. (2015). Two-level personas for nested design spaces. In Proceedings of the 33rd Annual ACM Conference on Human Factors in Computing Systems, pages 3265-3274.

Honey, P. and Mumford, A. (2006). The learning styles questionnaire: 80-item version. Peter Honey Publications Limited.

Knox, N. (2014). Persona empathy mapping. Cooper A blog about design, business, and the world we live in. http://www.cooper.com/journal/2014/5/persona-empathymapping. Acessado: 4/1/2016.

Moser, C. (2012). Child-centered game development (ccgd): developing games with children at school. Springer-Verlag London Limited, Pers Ubiquit Comput.

Rogers, Y., Sharp, H., and Preece, J. (2013). Design de interação - além da interação humano-computador.

Santos, V. M. (2013). Materiais audiovisuais para a educação a distância: a contribuição dos estilos de aprendizagem. Universidade Estadual Paulista (UNESP), Produção científica Faculdade de Ciências e Letras (FCLAR), Araraquara, SP.

Venkatesh, V., Morris, M. G., Davis, G. B., and Davis, F. D. (2003). User acceptance of information technology: Toward a unified view. pages 425-478. JSTOR.

Zaina, L. A. M. and Bressan, G. (2008). Classification of learning profile based on categories of student preferences. pages F4E-1-F4E-6. In: FIE - Frontiers in Education, New York. 38th Annual Frontiers in Education Conference.

Zaina, L. A. M., Bressan, G., Cardieri, M., and Rodrigues, J. F. (2012). e-lors: Uma abordagem para recomendacao de objetos de aprendizagem. volume 20, page 04 . Revista Brasileira de Informática na Educação (RBIE). 